\title{
CORRELATION BETWEEN EPITHELIAL-MESENCHYMAL TRANSITION AND COLLAGEN QUALITY IN ORAL DYSPLASIA AND SQUAMOUS CELL CARCINOMA
}

\author{
Nermeen S. Afifi", Rehab F. Fathi" and Shaimaa M. Masloub*
}

\begin{abstract}
Epithelial-mesenchymal transition (EMT) enhances the invasive ability, providing an explanation for the mechanism of tumor metastasis. The stiffness of the stromal extracellular matrix collagen can regulate the epithelial cell growth and migration in oral epithelial dysplasia (OED) and oral squamous cell carcinoma (OSCC).
\end{abstract}

Objectives: to study the epithelial expression of Vimentin as a marker for EMT and to elucidate the nature of collagen in OED and OSCC and finally to correlate EMT to collagen quality in these lesions.

Material and methods: the area fraction of immunohistochemical epithelial expression of Vimentin and color polarization of picrosirius red stained collagen fibers were detected in ten specimens of each grade of OED and OSCC as well as normal oral mucosa followed by statistical analysis.

Results: a statistically significant increase in the median area fractions of epithelial Vimentin expression and yellow-green fibers were detected as the grade of OED and OSCC progressed. Spearman's correlation coefficient revealed a statistically significant direct correlation between epithelial Vimentin expression and yellow-green fibers in these lesions.

Conclusion: there was a statistically significant increase in EMT and decrease in the collagen quality with the advance of OED and dedifferentiation of the neoplasm. The significant inverse correlation between EMT and collagen quality indicates the role of EMT in stromal collagen quality in these lesions and aid in their prognosis.

KEYWORDS: Vimentin, picrosirius red, polarized microscopy, collagen packing

* Lecturer of Oral Pathology, Faculty of Dentistry, Ain Shams University 


\section{INTRODUCTION}

Oral potentially malignant disorders was defined by the WHO in 2005 as the risk of malignancy being present in a lesion or condition either at time of initial diagnosis or at a future date ${ }^{(1)}$. These disorders are histopathologically reported as oral epithelial dysplasia (OED) and are graded as mild, moderate or severe. The grading system for OED most commonly used by histopathologists is the WHO criteria given in $1978^{(2)}$. The severe form of OED presents with a high risk to transform to oral squamous cell carcinoma (OSCC) which is composed of two discrete components i.e. the malignant epithelial cells and the stroma in which they are dispersed ${ }^{(3)}$. OSCC is the most prevalent malignancy in the oral cavity $^{(4)}$, a disease found particularly in low income communities and mainly a problem of older men, $90 \%$ of cases affecting $>45$ years age group who are exposed to the most common risk factors as tobacco and/or alcohol (IARC 2004) ${ }^{(5)}$.

During the development of OSCC, several changes occur in the epithelium; the breakdown of the basal lamina (BL) included ${ }^{(3)}$. BL is the barrier preventing the dysplastic epithelial cells from invading the stroma. It is synthesized, modified and secreted by epithelial and connective tissue cells ${ }^{(6}$ 7). Once the basal lamina is degraded by the altered epithelial cells, the invading neoplastic cells interact with the stroma, particularly the collagen component and this interplay is a crucial determinant of tumor progression and spread ${ }^{(8)}$.

Tumor metastasis is a complex dynamic process of multiple factors that pass through multi steps. Tumor invasion is the first implementation of metastasis ${ }^{(9)}$. Loss of epithelial morphology and uptake of mesenchymal characteristics, a process termed epithelial-mesenchymal transition (EMT), are typical transformations occurring in carcinoma cells and correlate with the local invasiveness and metastatic potential of the tumor ${ }^{(10)}$. EMT may lead to loss of adhesion among epithelial cells, as well as gaining the phenotypic characteristics of the interstitial cells, thus allowing the migration of tumor cells as well as enhancing the invasive ability, providing an explanation for the mechanism of tumor metastasis ${ }^{(9)}$.

Vimentin and E-cadherin are well identified markers for EMT associated with tumor progression (11). Vimentin is a cytoskeletal protein which is not expressed in normal epithelial cells, but is widely distributed in fibroblasts, endothelial cells and lymphocytes in the interstitial stroma. The abnormal expression of Vimentin was also observed in a variety of epithelial neoplasms and had close relationship with differentiation, invasion and metastasis of cancer cells ${ }^{(12)}$.

The tensile strength or stiffness of the stromal extracellular matrix collagen (EMC) in the connective tissue was found to affect and regulate the epithelial cell growth and migration in OED and OSCC as well. However, the dysplastic epithelialstromal interactions and nature of collagen fibers in the extracellular matrix of dysplastic epithelium has not yet been fully understood ${ }^{(13)}$.

The collagenous tissue, the "basic skeleton" of the stroma, undergoes extensive molecular as well as structural changes during the evolution and progression of carcinoma. It may benefit the tumor progression by inhibiting the host immune response. Furthermore, proteolysis or change in collagen composition can facilitate the mobilization of neoplastic cells within the stroma, subsequently aiding invasion and metastasis ${ }^{(14)}$. On the contrary, it can have antagonistic effects on tumor progression by resisting the tumor spread and motility of neoplastic cells within the stroma via inducing an abundant fibrous stroma (walling-off effect) ${ }^{(3)}$.

Traditional stains such as Van Gieson and trichrome stains, which are the combinations of two or more anionic dyes, are routinely used to histochemically detect collagen fibers in laboratory investigations. Since these methods lack precise selectivity, they are not ideal for collagen detection in light microscopy despite being technically 
simple to prepare ${ }^{(15)}$. Another disadvantage of these methods is that they fail to expose very thin collagen fibers which can further lead to underestimation of collagen content as they are usually overlooked (15). In order to overcome the potential problems encountered using these traditional stains, trials were first attempted by Constantine and Mowry in 1968 wherein a combination of Picrosirius Red (PSR) and polarization microscope was used for selective demonstration of collagen exposing precisely the previously missed thin collagen fibrils ${ }^{(16)}$.

Picrosirius Red stain is a combination of two anionic dyes i.e. Sirius Red F3BA (Direct Red 80) dissolved in a saturated picric acid solution. Sirius Red is a hydrophilic dye characterized by the presence of sulphonic acid groups. These groups react with basic groups present in the normal architecture of the collagen molecule ${ }^{(17)}$. There is a parallel arrangement of the dye molecules with the long axes of collagen fibers. This parallel relationship between the dye and collagen results in an enhanced birefringence which is an optical property of materials having a refractive index that depends on the polarization and propagation of the light passing through them. This birefringent property results in bright yellow to orange color of collagen when viewed under polarized light ${ }^{(18)}$.

The thickness as well as packing of the collagen fibers can be determined using PSR stain and visualized by polarizing microscopy. The examination of collagen fibers by this method helps to differentiate normally packed thick bundles of collagen fibers from pathologic collagen which is usually thinner, loosely packed and disorganized. Red polarization hue is indicative of thick fibers of closely packed collagen while greenish polarization hue is indicative of loosely packed thin pathologic collagen ${ }^{(19)}$. The alteration in stromal microenvironment around ED or neoplasms can predict the prognosis of the condition and is also useful in generating new therapeutic targets ${ }^{(13)}$.
In this work, we studied the epithelial expression of Vimentin as a marker for EMT in OED and OSCC and sought to determine if the specific pattern of Vimentin expression could be used to predict invasiveness. At the same time, we attempted to elucidate the nature of collagen in OED and OSCC, using PSR combined with polarizing microscopy and correlated EMT to collagen quality in these lesions.

\section{MATERIAL AND METHODS}

\section{Case selection}

Seventy formalin fixed paraffin-embedded archival blocks of OED and OSCC were obtained from the archives of the Oral Pathology Department, Faculty of Dentistry, Ain-Shams University. Ten specimens of normal mucosa were used as control. Ten specimens of each grade of OED and OSCC were selected.

\section{Immunohistochemical procedures}

For all specimens, $4 \mu \mathrm{m}$ sections were cut and mounted on positively charged glass slides. Sections were deparaffinized with xylene and rehydrated in graded ethyl alcohol. The sections were later immersed in citrate buffer solution of $\mathrm{pH} 4.8$ and put in the microwave oven before staining procedures.

For immunostaining, a universal kit (retrieved from Lab Vision) was used; peroxidase antiperoxidase method of immunostaining using the streptavidin biotin system was carried out and 3\% hydrogen peroxide was applied to the sections to block the endogenous peroxidase activity. Sections were immunostained using the concentrated primary monoclonal antibody (clone 56C6) against Vimentin (Thermo Fisher Scientific Laboratories, Ltd, United Kingdom), then incubated overnight at room temperature after rinsing with PBS (phosphate buffered saline) solution. Sections were then covered by the link antibody followed by the streptavidin labeling antibody; after rinsing with PBS, DAB chromogen was applied to the sections followed 
by counter stain. Finally, sections were dehydrated in graded alcohol, cleared in xylene and mounted. Immunohistochemical staining was carried out in Ain-Shams University Specialized Hospital.

\section{Procedures of PSR staining:}

This step was also carried out in Ain-Shams University Specialized Hospital. Paraffin-embedded tissue blocks were sectioned at $5 \mathrm{~mm}$ thickness; the sections were floated onto the slides. They were then incubated at $60 \mathrm{C}$ on the slide warmer for proper adhesion and later deparaffinized, hydrated and stained with PSR stain (Sigma-Aldrich, St. Louis, MO, USA) according to manufacturer instructions and viewed by polarizing microscopy.

\section{Assessment of immunohistochemical and PSR staining}

Four microscopic fields from each PSR stained slide presenting immunopositivity and also showing highest epithelial Vimentin immunopositivity were selected and photomicrographed at a magnification of (x 20) and (x 40). This was performed in the Precision Measurements Unit, Faculty of Dentistry, Ain-Shams University using a video camera (C5060, Olympus, Japan) which was mounted on a light microscope (BX60, Olympus, Japan). U-POT polarizing lens was used for examination of PSR stained sections. Images were then transferred to the computer system for analysis. Area fraction of Vimentin immunopositivity in epithelial cells only were measured. The area fraction of red-orange (RO) and yellow-green (YG) fibers in PSR stained slides were assessed. All the steps of image analysis were carried out using image analysis software (Image J, 1.41a, NIH, USA).

\section{Statistical analysis:}

Groups were compared using Kruskal Wallis test for non-parametric data followed by post-hoc analysis for pairwise comparisons. Spearman's correlation coefficient was used to determine the correlation between Vimentin and each of RO and YG fiber area \%. R Foundation for Statistical Computing, Vienna, Austria version 3.6.0 was used for the statistical analysis. Results were considered significant when $\mathrm{p}$-value $\leq 0.05$.

\section{RESULTS}

\section{Immunohistochemical results:}

Normal oral mucosa showed membranous and cytoplasmic immunoreactivity against Vimentin mainly in mesenchymal cells of the connective tissue, the epithelial cells were almost completely negative (Fig.1a). Positive, homogenous brown, epithelial immunostaining for Vimentin was found in all cases of the different grades of OED and OSCC examined in the present study. The reaction in OED is mainly membranous and cytoplasmic in scattered cells in the lower third of the epithelium as well as in mesenchymal cells in the underlying connective tissue (Fig.1b, c, d). The number of immunopositive cells appeared to increase steadily with the gradient of dysplasia.

On the other hand, OSCC showed Vimentin immunopositivity in scattered neoplastic epithelial cell in the cell nests and keratin pearls of well and moderately differentiated lesions with negative reaction in the keratin. The reaction is mainly membranous and cytoplasmic. The number of the immunopositive neoplastic epithelial cells appeared to increase in the poorly differentiated SCC (PDSCC). Stromal cells surrounding the neoplastic masses and individual cell showed intense Vimentin immunopositivity as well (Fig.1e, f, g). The lowest median area fraction for epithelial Vimentin expression was found in mild ED (3.51\%) while the highest Vimentin expression was found in PDSCC (33.81\%) as shown in (Table 1) and (Fig.3a).

Statistically, Kruskal Wallis test and multiple pairwise-comparison test showed significant differences between all groups except moderate ED versus mild ED and PDSCC versus moderately differentiated SCC (MDSCC) (Table 2). 


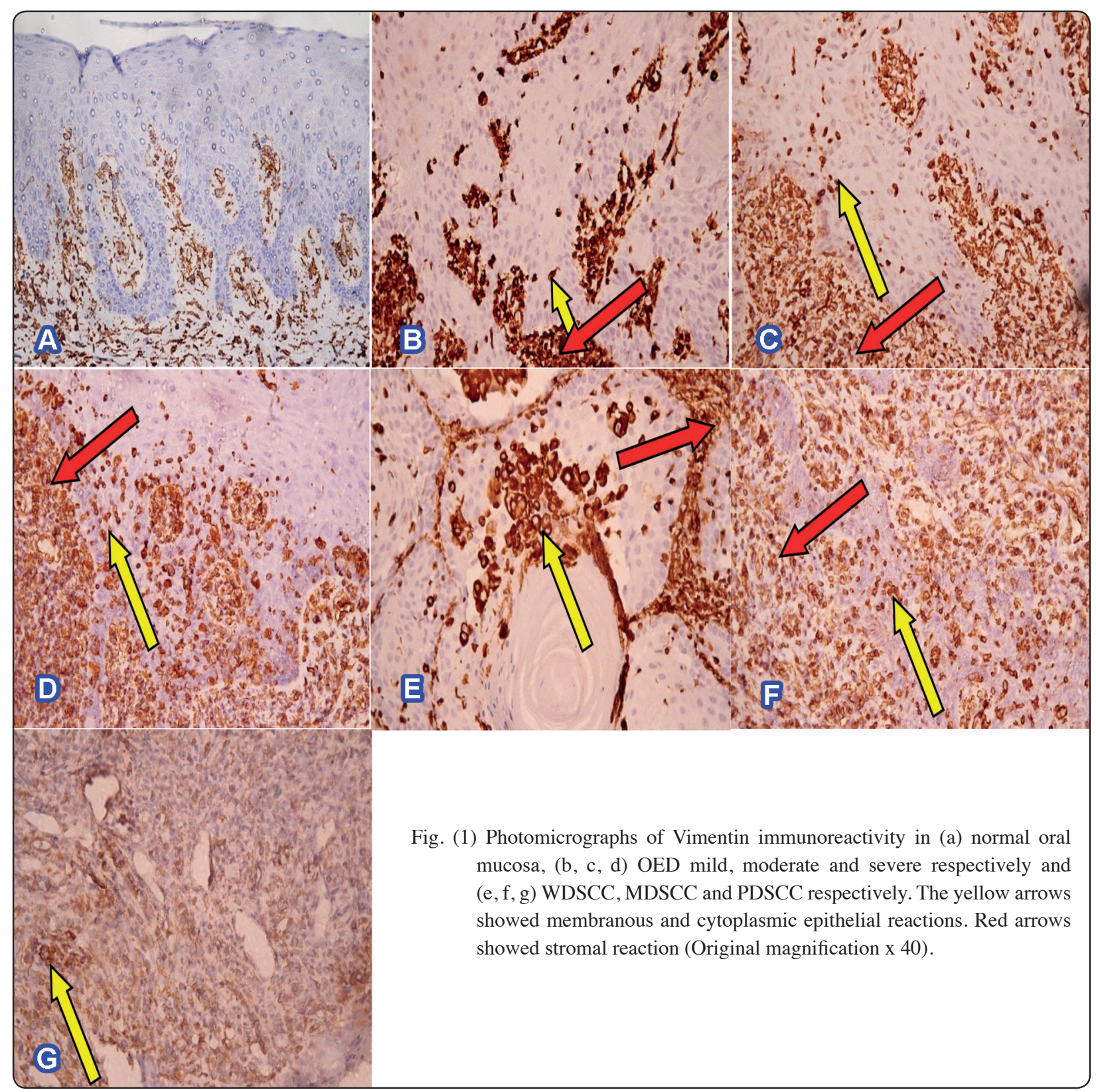

\section{PSR staining results}

PSR stained slides were examined using polarizing light microscope for collagen fibers quality and packing. Normal mucosa showed predominance of RO birefringence (Fig. 2a). As for OED, there was a predominance of $\mathrm{RO}$ hue in the mild and moderate dysplasia while the yellowish hue predominated up on progressing to severe ED (Fig. 2b, c, d). On the other hand, in OSCC, there was a gradual change in the polarizing colors from yellowish orange to YG fi- nally to intensified greenish hue on progressing from well differentiated SCC (WDSCC) to PDSCC (Fig. 2e, f, g). The highest median area fraction for RO fibers $(29.38 \%)$ and lowest median area fraction for YG fibers $(0.24 \%)$ was for normal oral mucosa followed by mild ED which recorded $25.86 \%$ and $1.04 \%$ for RO and YG fibers respectively. On the contrary, the lowest median area fraction for RO fibers (3\%) and the highest median area fraction for YG fibers (50.94\%) was for PDSCC (Table 1) (Fig. 3c). 


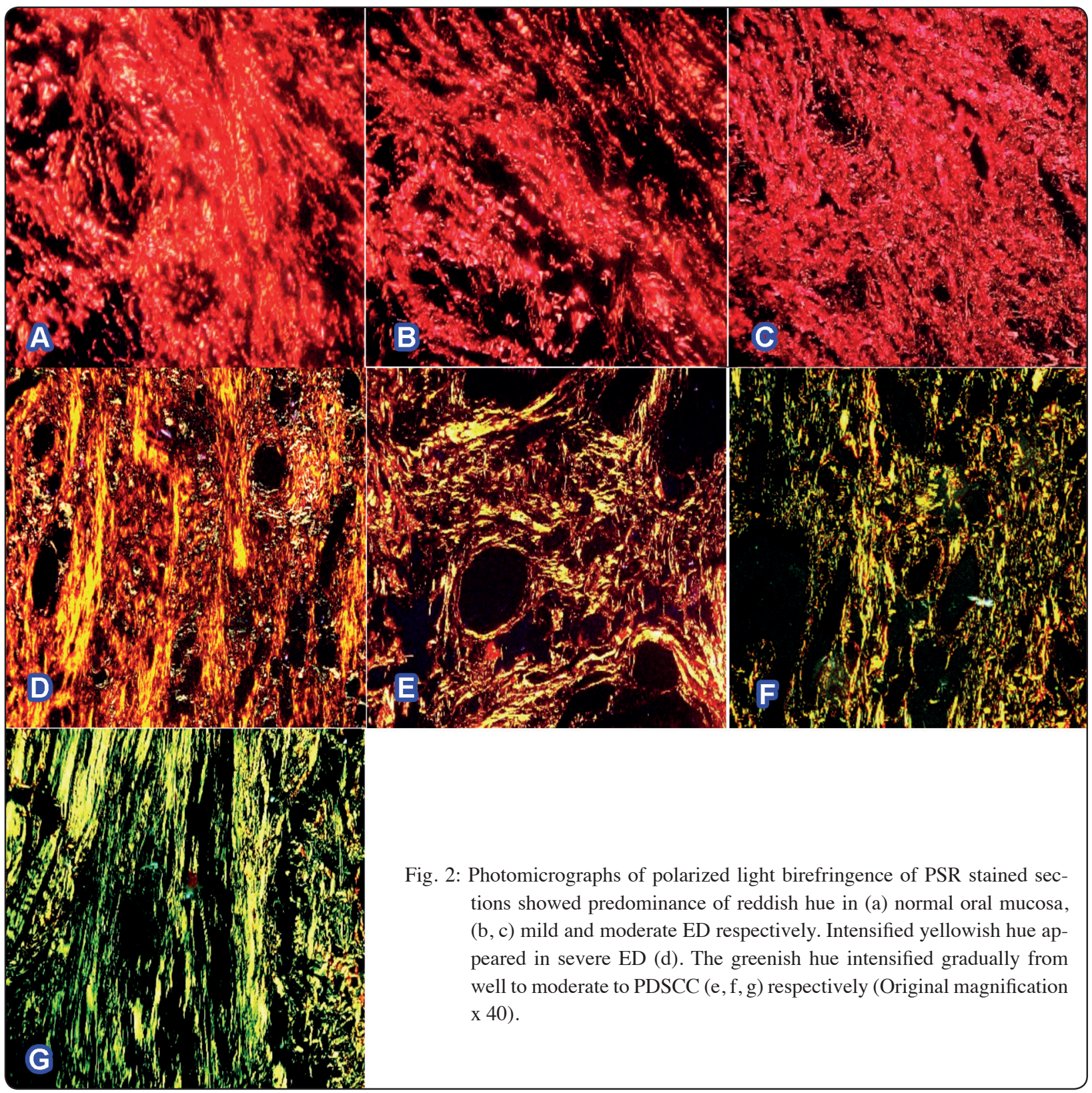

Statistically, Kruskal Wallis test and multiple pairwise-comparison test showed statistically significant differences between all groups except the following comparisons: mild ED Vs normal mucosa, mild ED Vs moderate ED, severe ED Vs WDSCC and finally, severe ED Vs MDSCC. Also, the three types of OSCC were not statistically different from each other (Fig. 3b) (Table 3). As regard the area fraction of YG birefringence, Kruskal Wallis test and multiple pairwise-comparison test showed significant differences between all groups except
MDSCC Vs severe ED, WDSCC Vs severe ED and finally, MDSCC Vs WDSCC (Table 3).

\section{Correlation between Vimentin, RO and YG fi- bers area \%}

Within each condition; there was no statistically significant correlation between Vimentin, RO and YG fiber area percentages except for well differentiated SCC where there was a statistically significant inverse correlation between Vimentin and $\mathrm{RO}$ area $\%(\varrho=0.636, \mathrm{P}$-value $=0.048)$. 
TABLE (1): Median area fraction of Vimentin epithelial immunoexpression, RO and YG fibers in different groups.

\begin{tabular}{|l|c|c|c|}
\hline \multicolumn{1}{|c|}{ Groups } & $\begin{array}{c}\text { Vimentin } \\
\text { Median (Range) }\end{array}$ & $\begin{array}{c}\text { RO fibers } \\
\text { Median (Range) }\end{array}$ & $\begin{array}{c}\text { YG fibers } \\
\text { Median (Range) }\end{array}$ \\
\hline Normal oral mucosa & --- & $29.38(22.77-33)$ & $0.24(0.09-0.78)$ \\
\hline Mild ED & $3.51(2.16-5.54)$ & $25.86(17.73-29.77)$ & $1.04(0.06-2.63)$ \\
\hline Moderate ED & $3.63(1.29-5.76)$ & $20.77(15.32-30.36)$ & $2.29(1.07-3.43)$ \\
\hline Severe ED & $9.76(5.5-16.74)$ & $8.73(6.25-11.32)$ & $33.02(29.76-41.23)$ \\
\hline WDSCC & $19.4(10.46-29.46)$ & $5.37(1.37-15.11)$ & $36.38(30.64-41.78)$ \\
\hline MDSCC & $30.12(17.24-40.52)$ & $4.45(2.66-8.74)$ & $39.43(30.77-43.43)$ \\
\hline PDSCC & $33.81(31.44-37.88)$ & $3(1.43-4.67)$ & $50.94(40.57-56.33)$ \\
\hline
\end{tabular}
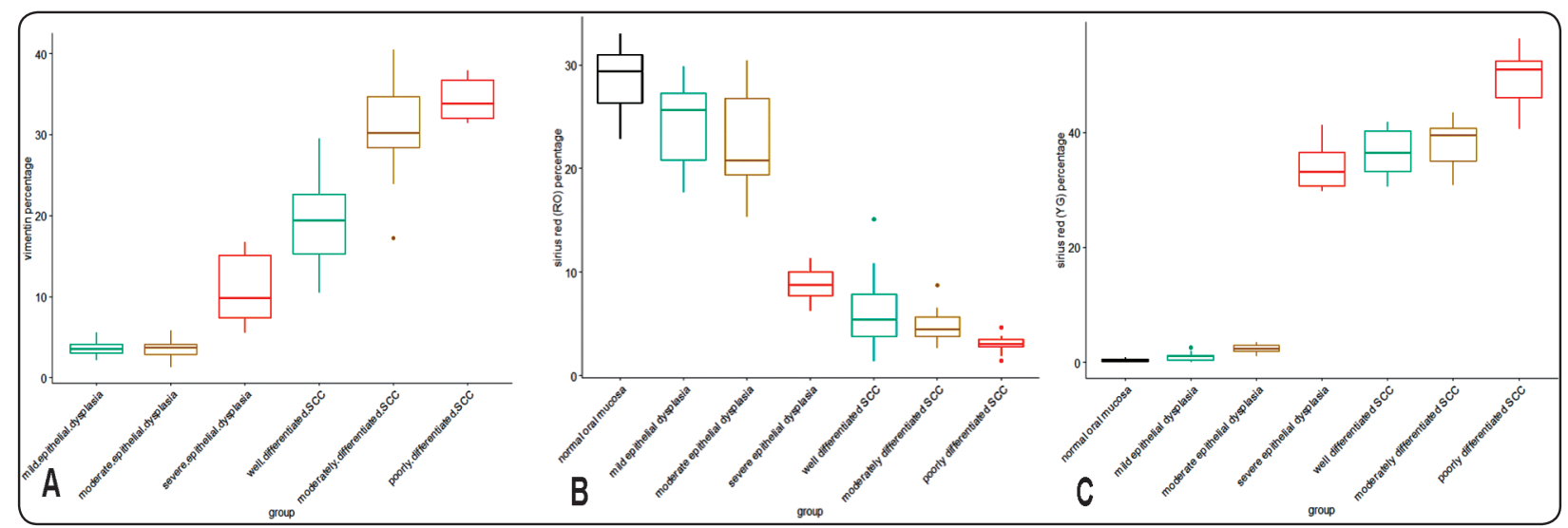

Fig. (3): Boxplots with error bars of (a) Vimentin immunoexpression, (b) RO PSR birefringence and (c) YG PSR birefringence in different groups.

TABLE (2) Multiple pairwise-comparison test for Vimentin expression between different groups.

\begin{tabular}{|c|c|c|}
\hline \multirow{4}{*}{ Group1 } & Group2 & P value \\
\hline \multirow{4}{*}{ Mild ED } & Moderate ED & 0.940 \\
\cline { 2 - 3 } & Severe ED & $<0.001^{*}$ \\
\cline { 2 - 3 } & WDSCC & $<0.001^{*}$ \\
\cline { 2 - 3 } & MDSCC & $<0.001^{*}$ \\
\cline { 2 - 3 } & PDSCC & $<0.001^{*}$ \\
\hline \multirow{3}{*}{ Moderate ED } & Severe ED & $<0.001^{*}$ \\
\cline { 2 - 3 } & WDSCC & $<0.001^{*}$ \\
\cline { 2 - 3 } & MDSCC & $<0.001^{*}$ \\
\cline { 2 - 3 } & PDSCC & $<0.001^{*}$ \\
\hline \multirow{3}{*}{ Severe ED } & WDSCC & $0.005^{*}$ \\
\hline \multirow{2}{*}{ WDSCC } & MDSCC & $<0.001^{*}$ \\
\hline & PDSCC & $<0.001^{*}$ \\
\hline & MDSCC & $0.005^{*}$ \\
\hline
\end{tabular}


TABLE (3) Multiple pairwise-comparison test for RO and YG birefringence between different groups.

\begin{tabular}{|c|c|c|c|c|c|c|}
\hline \multirow{2}{*}{ Group1 } & \multirow{2}{*}{ Group2 } & \multicolumn{4}{|c|}{ RO fibers } & \multirow{2}{*}{$\begin{array}{c}\text { YG fibers } \\
\text { P value }\end{array}$} \\
\hline & & Estimate & Conf. low & Conf. high & P value & \\
\hline \multirow{6}{*}{$\begin{array}{l}\text { Normal oral } \\
\text { mucosa }\end{array}$} & Mild ED & -4.110 & -8.835 & 0.615 & 0.129 & $0.040^{*}$ \\
\hline & Moderate ED & -6.190 & -10.915 & -1.465 & $0.003^{*}$ & $<0.001 *$ \\
\hline & Severe ED & -19.747 & -24.472 & -15.022 & $<0.001 *$ & $<0.001 *$ \\
\hline & WDSCC & -22.080 & -26.805 & -17.355 & $<0.001 *$ & $<0.001 *$ \\
\hline & MDSCC & -23.580 & -28.305 & -18.855 & $<0.001 *$ & $<0.001 *$ \\
\hline & PDSCC & -25.430 & -30.155 & -20.705 & $<0.001^{*}$ & $<0.001 *$ \\
\hline \multirow{5}{*}{ Mild ED } & Moderate ED & -2.080 & -6.805 & 2.645 & 0.830 & $0.005^{*}$ \\
\hline & Severe ED & -15.637 & -20.362 & -10.912 & $<0.001^{*}$ & $<0.001 *$ \\
\hline & WDSCC & -17.970 & -22.695 & -13.245 & $<0.001^{*}$ & $<0.001 *$ \\
\hline & MDSCC & -19.470 & -24.195 & -14.745 & $<0.001^{*}$ & $<0.001 *$ \\
\hline & PDSCC & -21.320 & -26.045 & -16.595 & $<0.001^{*}$ & $<0.001 *$ \\
\hline \multirow{4}{*}{ Moderate ED } & Severe ED & -13.557 & -18.282 & -8.832 & $<0.001^{*}$ & $<0.001 *$ \\
\hline & WDSCC & -15.890 & -20.615 & -11.165 & $<0.001 *$ & $<0.001 *$ \\
\hline & MDSCC & -17.390 & -22.115 & -12.665 & $<0.001 *$ & $<0.001 *$ \\
\hline & PDSCC & -19.240 & -23.965 & -14.515 & $<0.001^{*}$ & $<0.001 *$ \\
\hline \multirow{3}{*}{ Severe ED } & WDSCC & -2.333 & -7.058 & 2.392 & 0.741 & 0.195 \\
\hline & MDSCC & -3.833 & -8.558 & 0.892 & 0.188 & 0.058 \\
\hline & PDSCC & -5.683 & -10.408 & -0.958 & $0.009^{*}$ & $<0.001 *$ \\
\hline \multirow{2}{*}{ WDSCC } & MDSCC & -1.500 & -6.225 & 3.225 & 0.959 & 0.520 \\
\hline & PDSCC & -3.350 & -8.075 & 1.375 & 0.332 & $0.001 *$ \\
\hline MDSCC & PDSCC & -1.850 & -6.575 & 2.875 & 0.894 & $<0.001 *$ \\
\hline
\end{tabular}

*: Significant at $P \leq 0.05$

As regards the overall correlation (regardless of the condition); there was a statistically significant inverse correlation between Vimentin and $\mathrm{RO}$ area $\%(\mathrm{Q}=-0.825, \mathrm{P}$-value $<0.001)$. The larger Vimentin area $\%$, the smaller RO fiber area $\%$ and vice versa.
On the contrary, there was a statistically significant direct correlation between Vimentin and YG area \% ( $\varrho=0.847$, P-value <0.001). The larger Vimentin area $\%$, the larger YG fiber area $\%$ and vice versa (Table 4) (Fig. 4) 
TABLE (4): Results of Spearman's correlation coefficient for the correlation between area fraction \% of Vimentin, RO and YG fibers in all groups.

\begin{tabular}{|l|c|c|c|c|}
\hline \multirow{2}{*}{ Groups } & \multicolumn{2}{|c|}{ Vimentin and RO area $\%$} & \multicolumn{2}{|c|}{ Vimentin and YG area \% } \\
\cline { 2 - 5 } & $\begin{array}{c}\text { Correlation coefficient } \\
(\varrho)\end{array}$ & $\begin{array}{c}\text { Correlation } \\
\text { coefficient (@) }\end{array}$ & $P$-value \\
\hline Mild ED & -0.091 & 0.803 & 0.309 & 0.385 \\
\hline Moderate ED & -0.491 & 0.150 & 0.612 & 0.060 \\
\hline Severe ED & 0.224 & 0.533 & 0.139 & 0.701 \\
\hline WDSCC & 0.636 & $0.048^{*}$ & -0.455 & 0.187 \\
\hline MDSCC & 0.055 & 0.881 & 0.406 & 0.244 \\
\hline PDSCC & -0.115 & 0.751 & 0.079 & 0.829 \\
\hline Overall (Regardless of condition) & -0.825 & $<0.001 *$ & 0.847 & $<0.001^{*}$ \\
\hline
\end{tabular}

*: Significant at $P \leq 0.05$

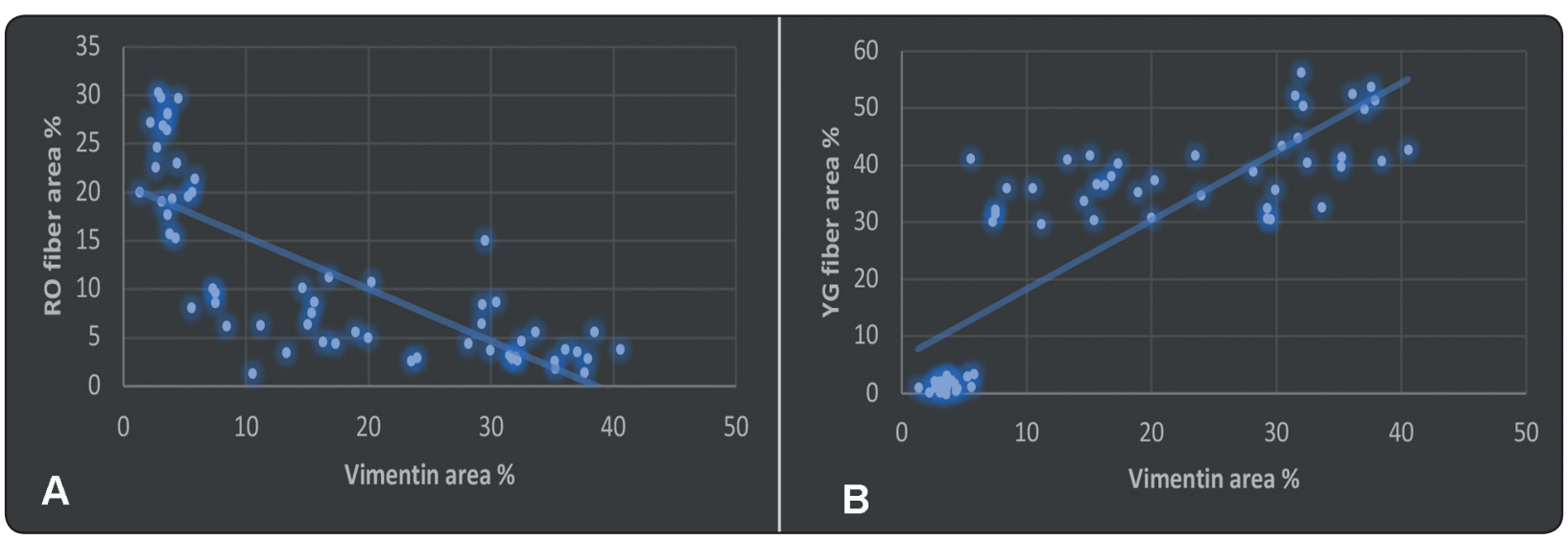

Fig. (4): Scatter diagrams representing inverse correlation between (a)Vimentin and RO fiber area \% and (b) direct correlation between Vimentin and YG fiber area \%, regardless of the group.

\section{DISCUSSION}

EMT is an important biological event in which cohesive and polarized epithelial cells switch to mesenchymal-like cells exhibiting no polarization, lack of cell-to-cell junctions and high mobility. This acquired migratory phenotype is important, particularly during tumor invasion and metastasis ${ }^{(20)}$. The altered expression of EMT markers such as E-cadherin, N-cadherin and Vimentin have recently been studied in various malignant epithelial tumors ${ }^{(21)}$.

Our study showed negative epithelial Vimentin immune-expression in normal oral mucosa while the different grades of OED and OSCC showed positivity of different densities. We observed a statistically significant increase in epithelial Vimentin immune-expression with progression from mild ED to PDSCC. 
The results of the present study were in agreement with the argument of Fernández et al. ${ }^{(12)}$ and Akhtar et al. ${ }^{(22)}$. Another study by Wang et al. ${ }^{(23)}$ reported increased Vimentin expression at the invasive front of OSCC when compared to central/superficial areas of the tumor. The same happens in prostate, gastric, colorectal, mammary and cervical/uterine neoplasms in several reported findings where this over expression is associated with poorly differentiated tumors with an invasive phenotype ${ }^{(24,25)}$.

Vimentin is an intermediate filament normally expressed by cells presenting a mesenchymal phenotype ${ }^{(26)}$. Its expression by epithelial malignant tumors cells is associated with an alteration in their behavior with increase in their motility, high invasiveness and potency to spread within the surrounding stroma. Therefore, it has been considered a reliable EMT marker ${ }^{(21)}$. In vitro studies have shown that inhibition of Vimentin expression results in reversal of the mesenchymal phenotype with reduction of migratory and invasive capabilities of OSCC cell lines ${ }^{(27)}$. In fact, Vimentin expression has been associated with higher prevalence of lymph node metastasis in esophageal ${ }^{(28)}$ as well as in head and neck SCC ${ }^{(29)}$. Moreover, Liu et al. ${ }^{(30)}$ reported a higher prevalence of recurrences and a less survival time in OSCC showing positive Vimentin expression. Gain of Vimentin is a hallmark of tumor progression and its expression generally indicates a poor prognosis ${ }^{(20)}$.

OSCC is usually preceded with ED which is characterized by the anaplastic changes, morphologically and structurally, in stratified squamous epithelial cells ${ }^{(13)}$. SCC consists of two interdependent components - the neoplastic epithelial cells and the stroma. These components interact on a regular basis and to an extent that discrepancy in one could affect the other ${ }^{(31)}$. Hence, for a better prediction of course and outcome of SCC, both should be given equal weightage in the evaluation/grading system. Although the epithelial component of OSCC has been studied extensively, the stroma is still a less explored arena. Among the stromal components, only the inflammatory component has been included in few of the grading systems for SCC ${ }^{(32)}$. The other stromal constituents, i.e., the collagen, elastic and reticulin fibers still await to receive a recognition as important factors in the progression of this disease ${ }^{(33)}$. Collagen, one of the major elements of the stroma, is primarily affected by the stromal changes affected by the altered epithelial cells at the site of tumor cell invasion. Although numerous studies have been conducted on the relationship between the stromal reaction and the prevalence of OSCC, research regarding the early stromal reaction in oral potentially malignant disorders is fragmentary ${ }^{(34)}$. Thus, the present study assessed the collagen fiber changes with progressive grades of OED and OSCC.

In this work, normal oral mucosa showed predominantly RO birefringence indicating predominance of a well-packed mature type of collagen (collagen type I). As for ED, there was a gradual change in the polarizing colors from reddish orange to slight increase in the yellowish tinge to finally reach an intensified yellowish hue on progressing from mild ED to severe ED.

On the other hand in SCC, there was a gradual change in the polarizing colors from yellowish orange to yellowish green to finally reach an intensified greenish hue on progressing from well to poorly differentiated SCC. This gradual change in the birefringence color, indicates a gradual decrease in the collagen quality, maturity and packing as the grade of ED or the grade of malignancy increases. Our results were in accordance with the results of studies conducted by Sharma et al. (3) and several others ${ }^{(35-39)}$.

The changes in birefringence color observed in the current study could be explained by the argument stated by Allon et al. ${ }^{(40)}$, who suggested that when collagen was stained with PSR and viewed by polarized light microscopy, thin collagen fibers (type III) showed green to greenish yellow birefringence. Moreover, thick collagen fibers (type I) ranged from yellowish orange to orange red birefringence. The 
green to greenish yellow color of both thin and thick fibers was explained by the idea that the collagen was then loosely packed, while orange red color originated from tightly packed fibers ${ }^{(40)}$. Thus, the particular colors produced by polarization microscopy of PSR stained section could be examined to define the fiber size, alignment and packing, crosslinking of the fiber bundles, interstitial ground substance as well as the water content ${ }^{(41)}$.

In addition, it was reported that as the grade of OSCC progressed, the packing of collagen fibers decreased and the orientation of collagen fibers changed from parallel to haphazard. This could possibly be due to some enzymatic degeneration of the existing collagen or due to the formation of new abnormal/pathologic collagen. Moreover, it could also be the result of increased amount of type III collagen which occurs singly rather than in bundles like Type I collagen ${ }^{(38)}$. Similarly, Martins et al. ${ }^{(42)}$ in their study on OSCC observed that in a lower grade of malignancy, collagen fibers near invasive front were arranged parallel to the tumor islands. However, in a higher grade of malignancy, the ECM exhibited lower levels of collagen synthesis and the fibers were irregular, disorganized and dissociated ${ }^{(42)}$. Linear with that, George et al. ${ }^{(43)}$ in their study evaluated the response of stroma in varying grades of OSCC and observed that as the grade of carcinoma progressed, the amount of collagen decreased.

Furthermore, the change in the birefringence of thick fibers in the present study could also be explained by the change in the collagen content of the stroma due to adjacent tumor cells which secrete enzymes such as collagenases or MMPs; or the disorganized stroma and altered matrix secreted by the proliferating undifferentiated tumor cells ${ }^{(44)}$.

The responsibility of EMT for the changes in collagen quality also observed in this study, is still a matter of debate. During the last decade, several research groups agreed with the possibility of occurrence of EMT during organ fibrosis with subsequent appearance of epithelial-derived myofibroblasts causing tissue fibrosis ${ }^{(45-47)}$. On the other hand, this concept has been challenged by other studies, suggesting that myofibroblasts are exclusively derived from sources other than epithelial cells ${ }^{(48-49)}$.

Several studies have shown an increase in the level of COL1A1 and COL3A1 gene expression in renal ${ }^{(50)}$ as well as in lung ${ }^{(51)}$ epithelial cells after EMT induction. In addition, emerging evidence suggests that a major source of fibrosis as well as tumor-associated myofibroblasts is through transdifferentiation from non-malignant epithelial cells or epithelial-derived carcinoma cells through EMT ${ }^{(52)}$. Furthermore, Gabbiani et al. ${ }^{(53)}$ suggested the association between the appearance of large numbers of myofibroblasts and replacement of collagen type I by collagen type III during wound healing. Furthermore, they proposed that myofibroblasts, in addition to their contractile activity, synthetize type III collagen.

Moreover, the study of Ehrlich and Hunt (54) proved the inability of myofibroblasts to produce thick and compact collagen fibers in pathological conditions. Thus, we could explain the statistically significant direct correlation between Vimentin epithelial immunoexpression and the area fraction of yellowish-green fibers observed in this study by the suggested responsibility of EMT for the low quality of collagen synthesized by epithelial-derived myofibroblasts in the stroma of OED and OSCC. This correlation, in turn, explains the progression of the neoplasm, therefore predicting the prognosis of the tumor.

At the end of this study, we concluded that there was a statistically significant increase in EMT and decrease in the collagen quality with the advance of OED and dedifferentiation of the neoplasm. The significant inverse correlation between EMT and collagen quality indicates the role of EMT in stromal collagen quality in these lesions and aid in their prognosis. 


\section{REFERENCES}

1. George A.: Potentially malignant disorders of oral cavity. Oral Maxillofac Pathol J. 2011; 2: 95-100.

2. Kardam P., Rehani S., Mehendiratta M., Sahay K., Mathias Y. and Sharma R.: Journey of leukoplakia so far - an insight on shortcomings of definitions and classifications. J Dent Oral Disord Ther. 2015; 3: 1-6.

3. Sharma R., Rehani S., Mehendiratta M., Kardam P., Kumra M., Mathias Y., Yadav J. and Sahay K.: Architectural analysis of picrosirius red stained collagen in oral epithelial dysplasia and oral squamous cell carcinoma using polarization microscopy. J Clin and Diag Res. 2015; 9: 13-16.

4. Cao G. and Li Z.: A single nucleotide polymorphism in the matrix metalloproteinase-1 promoter enhances oral squamous cell carcinoma susceptibility in a Chinese population. Oral Oncol. 2006; 42: 32-8.

5. Scully C. and Bagan J.: Oral squamous cell carcinoma: overview of current understanding of aetiopathogenesis and clinical implications. Oral Dis. 2009; 15: 388-99.

6. Engbring A. and Kleinman K.: The basement membrane matrix in malignancy. J Pathol 2003; 200: 465-70.

7. Williams K.: Molecular pathogenesis of oral squamous carcinoma. Mol Pathol 2000; 53: 165-72.

8. Yokoyama M.: Alterations in stromal reaction during tumor progression in oral mucosa. J Hard Tissue Biology. 2011; 20: 23-30.

9. Zhou J., Tao D., Xu Q., Gao Z. and Tang D.: Expression of E-cadherin and vimentin in oral squamous cell carcinoma. Int J Clin Exp Pathol 2015; 8: 3150-3154.

10. Hollier B., Evans K. and Mani S.: The epithelial-tomesenchymal transition and cancer stem cells: a coalition against cancer therapies. J Mammary Gland Biol Neoplasia. 2009; 141:29-43.

11. Natarajan J, Chandrashekar C. and Radhakrishnan R. Critical biomarkers of epithelial-mesenchymal transition in the head and neck cancers. J Cancer Res Ther. 2014; 10: $512-518$.

12. Fernández A., Marshall M., Santibañez N., Martínez C., Fernández J., Martínez R., Briceño J., Zumaran C., Peñaloza J., Fuentevilla I. and Haida Z.: Comparing the immuno-expression of endothelial markers in normal oral mucosa, oral epithelial dysplasia and oral squamous cell carcinoma: relationship between e-cadherin, vimentin, cd31, cd117 and epithelial-mesenchymal transition. MOJ Anat Physiol. 2017; 4:301-306.

13. Varghese S., Sarojinis., George G., Vinod S., Mathew P., Babu A. and Sebastian J.: Evaluation and comparison of the biopathology of collagen and inflammation in the extracellular matrix of oral epithelial dysplasia and inflammatory fibrous hyperplasia using picrosirius red stain and polarising microscopy: a preliminary study. $\mathrm{J}$ cancer prev. 2015; 20: 275-280.

14. Fuentes B., Duaso J., Droguett D., Castillo C., Donoso W., Rivera C., Venegas B. and Kemmerling U.: Progressive extracellular matrix disorganization in chemically induced murine oral squamous cell carcinoma. International Scholarly Research Network ISRN Pathology. 2012; Article ID 359421, 7 pages.

15. Hadi A., Mouchaers K., Schalij I., Grunberg K., Meijer G., Vonk-Noordegraaf A. and Beliën J. Rapid quantification of myocardial fibrosis: a new macro-based automated analysis. Cell Oncol (Dordr). 2011; 34: 343-344.

16. Constantine V. and Mowry R.: Selective staining of human dermal collagen II. The use of picrosirius red F3BA with polarization microscopy. J Invest Dermatol. 1968; 50: 419-23.

17. Junqueira L., Bignolas G. and Brentani R.: Picrosirius staining plus polarization microscopy, a specific method for collagen detection in tissue sections. Histochem J. 1979; 11: 447-55.

18. Montes G. and Junqueira L.: The use of the Picrosiruspolarization method for the study of the biopathology of collagen. Mem Inst Oswaldo Cruz. 1991; 86: 1-11.

19. de Paula A., Carvalhais J., Domingues M., Barreto D. and Mesquita R.: Cell proliferation markers in the odontogenic keratocyst: effect of inflammation. J Oral Pathol Med 2000; 29: 477-82.

20. Scanlon C., van Tubergen E., Inglehart R. and D'Silva N.: Biomarkers of epithelial-mesenchymal transition in squamous cell carcinoma. J Dent Res. 2013; 92: 114-21.

21. Zeisberg M. and Neilson E.: Biomarkers for epithelial-mesenchymal transitions. J Clin Invest. 2009; 119: 1429-37.

22. Akhtar K.,Ara A., Siddiqui S. and Sherwani R.: Transition of Immunohistochemical Expression of E-Cadherin and Vimentin from Premalignant to Malignant Lesions of Oral Cavity and Oropharynx. Oman Medical Journal. 2016; 31 : 165-169. 
23. Wang C., Huang H., Huang Z., Wang A., Chen X., Huang L. Zhou X. and Liu X.: Tumor budding correlates with poor prognosis and epithelial mesenchymal transition in tongue squamous cell carcinoma. J Oral Pathol Med. 2011; 40: 545-55.

24. Satelli A. and Li S.: Vimentin as a potential molecular target in cancer therapy or Vimentin, an overview and its potential as a molecular target for cancer therapy. Cell Mol Life Sci. 2011; 68: 3033-3046.

25. Costa L., Leite C., Cardoso S., Loyola A., Faria P., Souza P. and Horta M.: Expression of epithelial mesenchymal transition markers at the invasive front of oral squamous cell carcinoma. J Appl Oral Sci. 2015; 23:169-178.

26. Satelli A. and Li S.: Vimentin in cancer and its potential as a molecular target for cancer therapy. Cell Mol Life Sci. 2011; 68: 3033-3046.

27. Paccione R., Miyazaki H., Patel V., Waseem A., Gutkind J., Zehner Z. and Yeudall W.: Keratin down-regulation in Vimentin-positive cancer cells is reversible by vimentin RNA interference, which inhibits growth and motility. Mol Cancer Ther. 2008; 7: 2894-2903.

28. Jin H., Morohashi S., Sato F., Kudo Y., Akasaka H., Tsutsumi S., Ogasawara H., Miyamoto K., Wajima N., Kawasaki H., Hakamada K. and Kijima H.: Vimentin expression of esophageal squamous cell carcinoma and its aggressive potential for lymph node metastasis. Biomed Res. 2010; 31: 105-112.

29. Mandal M., Myers J., Lippman S., Johnson F., Williams M., Rayala S., Ohshiro K., and El-Naggar A.: Epithelial to mesenchymal transition in head and neck squamous carcinoma: association of Src activation with E-cadherin down-regulation, Vimentin expression, and aggressive tumor features. Cancer. 2008; 112: 2088-2100.

30. Liu L., Jiang X., Zhou X., Wang D., Song X. and Jiang H.: Up regulation of Vimentin and aberrant expression of E-cadherin/ beta-catenin complex in oral squamous cell carcinomas: correlation with the clinicopathological features and patient outcome. Mod Pathol. 2010; 23: 213-24.

31. Bissell M. and Radisky D.: Putting tumors in context. Nat Rev Cancer. 2001; 1: 46-54

32. Sawazaki-Calone I., Rangel A., Bueno A., Morais C., Nagai H., Kunz R., Souza R., Rutkauskis L., Salo T., Almangush A. and Coletta R.: The prognostic value of histopathological grading systems in oral squamous cell carcinomas. Oral Dis. 2015; 21: 755-61.
33. Patricia S., Suzigan S., Carvalho H. and Taboga S.: Structural characterization and distribution of elastic system fibers in the human prostate and some prostatic lesions. Braz J Morphol Sci. 2003; 20: 101-107.

34. Koontongkaew S.: The tumor microenvironment contribution to development, growth, invasion and metastasis of head and neck squamous cell carcinomas. J Cancer. 2013; 4: 66-83.

35. Kalele K., Managoli N., Roopa N., Kulkarni ., Bagul N. and Kheur S.: Assessment of collagen fiber nature, spatial distribution, hue and its correlation with invasion and metastasis in oral squamous cell carcinoma and surgical margins using Picro Sirius red and polarized microscope. J Dent Res Rev. 2014; 1: 14-17.

36. Manjunatha B., Agrawal A. and Shah V.: Histopathological evaluation of collagen fibers using picrosirius red stain and polarizing microscopy in oral squamous cell carcinoma. J Can Res Ther. 2015; 11: 272-276.

37. Venigella A. and Charu S.: Evaluation of collagen in different grades of oral squamous cell carcinoma by using the picrosirius red stain -Histochemical study. J Clin Diagn Res. 2010; 4: 3444-3449.

38. Kardam P., Mehendiratta M., Rehani S., Kumra M., Sahay K. and Jain K.: Stromal fibers in oral squamous cell carcinoma: A possible new prognostic indicator? J Oral Maxillofac Pathol. 2016; 20: 405-412.

39. Gopinathan P., Kokila G., Jyothi M., Ananjan C., Pradeep L. and Nazir S.: Study of collagen birefringence in different grades of oral squamous cell carcinoma using picrosirius red and polarized light microscopy. Scientifica (Cairo). 2015; 2015:802980.

40. Allon I., Vered M., Buchner A. and Dayan D.: Stromal differences in salivary gland tumors of a common histopathogenesis but with different biological behavior: a study with picrosirius red and polarizing microscopy. Acta Histochemica. 2006; 108: 259-264.

41. Koren R., Yaniv E., Kristt D., Shvero J., Veltman V., Grushko I., Feinmesser R., Sulkes J. and Gal R.: Capsular collagen staining of follicular thyroid neoplasms by picrosirius red: role in differential diagnosis. Acta Histochemica. $2001 ; 103: 151-157$.

42. Martins G., Reis S. and Silva T.: Collagen type I expression in squamous cell carcinoma of the oral cavity. Pesqui Odontol Bras 2003; 17: 82-88. 
43. George J., Narang R. and Rao N.: Stromal response in different histological grades of oral squamous cell carcinoma: A histochemical study. Indian J Dent Res. 2012; 23: 842.

44. Woolgar J.: Histopathological prognosticators in oral and oropharyngeal squamous cell carcinoma. Oral Oncol. 2006; 42: 229-239.

45. Iwano M., Plieth T., Danoff C., Xue H. and Neilson E.: Evidence that fibroblasts derive from epithelium during tissue fibrosis. J Clin Invest. 2002; 110: 341.

46. Tanjore X., Xu V., Polosukhin A., Degryse B., Han T., Sherrill D., Plieth E., Neilson T. and Lawson W.: Contribution of epithelial-derived fibroblasts to bleomycin-induced lung fibrosis. Am J Respir Crit Care Med 2009; 180: 657-665.

47. Norihiko S. and Andrew M.: Fibrosis of two: Epithelial cell-fibroblast interactions in pulmonary fibrosis. Biochimica et Biophysica Acta. 2013; 1832: 911-921.

48. Koesters R., Kaissling B., Lehir M., Picard N., Theilig F., Gebhardt R., Glick A., Hähnel B., Hosser H., Gröne H. and Kriz W.: Tubular overexpression of transforming growth factor-beta1 induces autophagy and fibrosis but not mesenchymal transition of renal epithelial cells. Am J Pathol. 2010; 177: 632-643.
49. Humphreys B., Lin S., Kobayashi A., Hudson T., Nowlin B., Bonventre J., Valerius M., McMahon A. and Duffield J.: Fate tracing reveals the pericyte and not epithelial origin of myofibroblasts in kidney fibrosis. Am J Pathol. 2010; 176: 85-97.

50. Doerner A. and Zuraw B.: TGF-beta1 induced epithelial to mesenchymal transition (EMT) in human bronchial epithelial cells is enhanced by IL-1beta but not abrogated by corticosteroids. Respir Res. 2009;10: 100 .

51. Li J., Zhu H., Huang X., Lai K., Johnson R. and Lan H.: Smad7 inhibits fibrotic effect of TGF-Beta on renal tubular epithelial cells by blocking Smad2 activation. J Am Soc Nephrol. 2002; 13: 1464-1472.

52. Derek R., Paraic K. and Mina B.: Fibrosis and cancer: Do myofibroblasts come also from epithelial cells via EMT? J Cell Biochem. 2007; 101: 830-839.

53. Gabbiani G., Lous M., BaileyA., Bazin S. and Delaunay A.: Collagen and myofibroblasts of granulation tissue. Virchows Arch. 1976; 21: 133-145.

54. Ehrlich H. and Hunt T.: Collagen organization critical role in wound contraction. Adv. Wound Care. 2012; 1: 3-9. 